\title{
Porteurs de cocaïne asymptomatiques aux urgences
}

\section{Asymptomatic Cocaine Body Packers in the Emergency Department}

\author{
D. Douillet · A. Fieni · B. Carneiro
}

Reçu le 14 octobre 2018; accepté le 24 janvier 2019

(C) SFMU et Lavoisier SAS 2019
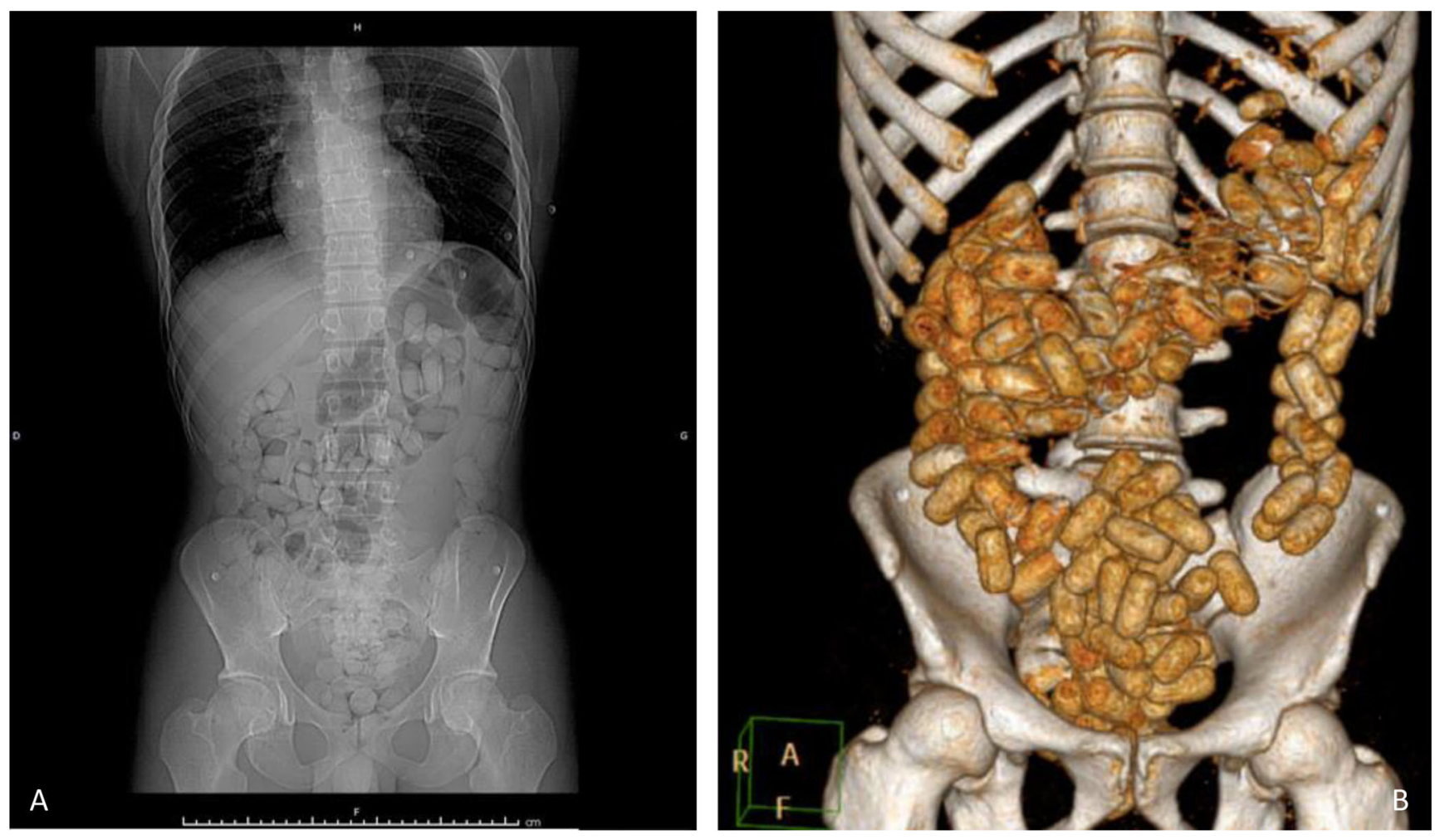

Fig. 1 A. Abdomen sans préparation standard mettant en évidence des images hyperdenses oblongues dans le plan frontal (signe $\mathrm{du}$ « tic-tac »). B. Tomodensitométrie thoracique inférieure et abdominopelvienne avec images de reconstruction montrant la présence d'environ 130 corps étrangers oblongs de $30 \times 20 \mathrm{~mm}$ répartis sur le cadre colique sans signe de complication

La prise en charge des patients suspectés d'ingestion de capsules de drogues est mal codifiée et parfois complexe. Un patient âgé de 19 ans est admis par les services douaniers

D. Douillet $(\bowtie) \cdot$ A. Fieni $\cdot$ B. Carneiro

Département de médecine d'urgence,

centre hospitalier universitaire d'Angers,

4, rue Larrey, F-49100 Angers, France

e-mail : delphine.douillet@chu-angers.fr

D. Douillet

UFR santé, université d'Angers,

F-49100 Angers, France aux urgences pour suspicion d'ingestion de capsules de cocaïne. Il est d'origine guyanaise et est arrivé en France depuis trois jours. Il a été appréhendé dès son arrivée par les services douaniers. Il n'a pas d'antécédent particulier ni de traitement au long cours. À son admission aux urgences, sa pression artérielle est à 130/83 mmHg, la fréquence cardiaque à 73 battements/minute, la saturation en oxygène en air ambiant est à $100 \%$. L'examen clinique retrouve un abdomen météorisé souple indolore sans signe d'occlusion. Il ne présente aucun signe d'intoxication à la cocaïne. L'électrocardiogramme est normal. Le bilan biologique ne retrouve pas d'anomalie. Une radiographie de type abdomen sans 
préparation (ASP) est réalisée montrant un grand nombre de corps étrangers (Fig. 1). En complément, une tomodensitométrie thoracique inférieure et abdominale confirme la présence de 130 corps étrangers. Le risque d'occlusion, de perforation digestive et d'intoxication à la cocaïne est jugé élevé en regard du nombre important de capsules de cocaïne, et une admission en unité de surveillance continue est décidée. Le patient est alors traité par polyéthylène glycol per os. L'évolution est simple sans manifestation toxique et avec une exonération de l'ensemble des capsules en trois jours. Les capsules sont remises aux autorités. En l'absence de contre-indication, le patient est placé en garde à vue. En l'espace de trois semaines, trois patients seront admis aux urgences dans le même contexte et avec un nombre élevé de capsules ingérées. Leurs prises en charge diagnostiques et thérapeutiques seront toutes identiques.

Le premier body packer décrit date de 1973 à Toronto et avait présenté une occlusion intestinale après l'ingestion de haschich conditionné dans un préservatif. Trente-cinq ans plus tard, la prise en charge de ces patients reste mal définie. Lors d'une suspicion, la réalisation d'un ASP est recommandée en première intention [1]. Sa sensibilité diagnostique est estimée entre 85 et $90 \%$ [2]. Il existe trois principaux signes pour identifier les capsules. Le signe du « tic-tac », le plus sensible, se réfère à l'identification d'opacités oblongues, uniformes, homogènes dans le tractus gastro-intestinal, comme dans le cas décrit. Le signe du «double condom » fait référence à un croissant d'air emprisonné entre les couches de latex ou l'emballage en cellophane. Enfin, le « signe de la rosette » fait référence à l'apparition de particules d'air piégées dans le nœud noué à la fin de l'emballage du toxique. L'estimation du nombre et de la localisation des sachets est importante tant sur le plan clinique que légal, compte tenu des complications potentiellement mortelles de cette ingestion. L'ASP sous-estime cependant la quantité de capsules ingérées comparativement au scanner abdominal [2]. Le scanner abdominal faible dose apparaît être une alternative plus fiable (sensibilité allant de 96 à $100 \%$ ) majorant peu l'irradiation reçue. Chez les patients asymptomatiques, la surveillance en milieu de soins intensifs est recommandée. Le taux de complications, définies par la survenue d'une occlusion digestive ou d'un syndrome d'intoxication à la cocaïne par perforation de capsule, est variable selon les études allant de 1,4 à $5 \%$.

\section{Références}

1. Traub SJ, Hoffman RS, Nelson LS (2003) Body Packing — The internal concealment of illicit drugs. N Engl J Med 349:2519-26

2. Esterson YB, Patel V, Nicastro J, Friedman B (2017) Plain radiography may underestimate the burden of body packer ingestion: a case report. Clin Imaging 44:57-60 